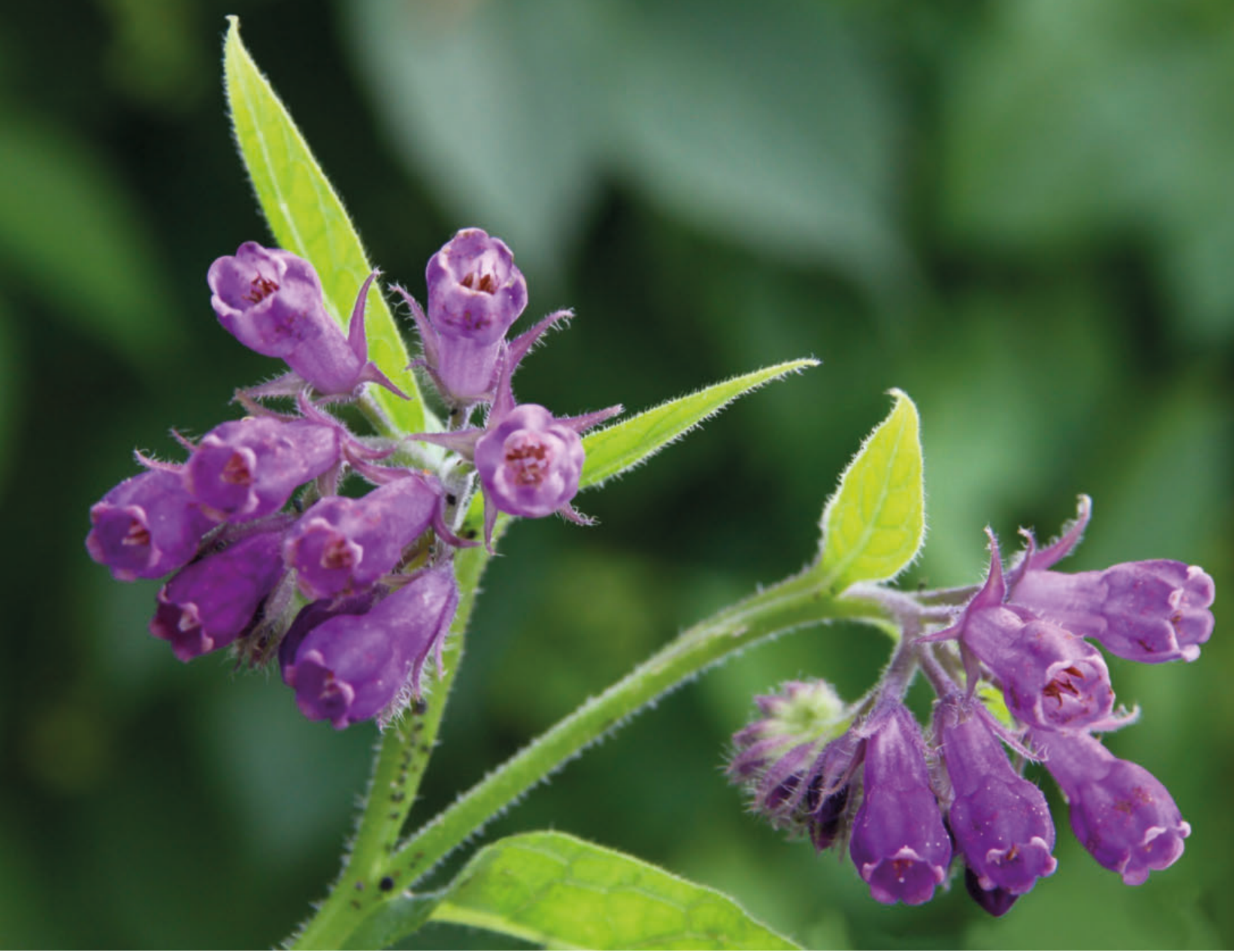

Beinwell. Foto: Maria Brzostowska/Adobe Stock

\title{
Phytotherapie bei Rheuma
}

Wirkstoffe wie Ibuprofen oder Diclofenac hemmen Entzündungen bei Rheuma Phythotherapeutika zeigen ähnliche Wirkungen

Roman Huber

Phytotherapie wird sowohl von Patienten mit Arthrose wie auch bei entzündlichem Rheuma häufig eingesetzt. Zur Anwendung kommt sie äußerlich, z.B. als Salbe oder Essenz, in oraler Form als Fertigpräparat oder Tee, aber auch als subkutane/periartikuläre Injektion.

Unter den Stichworten „Phytotherapy“ und „Arthritis“ finden sich in der Datenbank PubMed 1034 Treffer, 187 davon betreffen klinische Studien. Insbesondere zu chinesischen Pflanzen und Kräutermischungen gibt es eine hohe Anzahl von In-vitro-Untersuchungen und Tierversuchen, die eine Wirkung bei rheumatischen Erkrankungen vermuten lassen. Diese sind jedoch, wie z.B. Extrakte aus der Dreiflügelfrucht (Trypterigium wilfordii), in Europa zumeist nicht erhältlich oder aufgrund möglicher Toxizität problematisch und werden daher in dieser Übersicht nicht behandelt.

\section{Wirkmechanismus}

Phytotherapeutika bzw. deren Inhaltsstoffe verfügen über Wirkmechanismen, die bei rheumatischen Erkrankungen plausibel sind.
Häufiges Wirkprinzip ist die Modifikation des Eicosanoidstoffwechsels (Prostaglandine, Leukotriene) durch die Hemmung von Cyclooxigenasen (COX) bzw. Lipoxigenasen (LOX). COX bzw. LOX katalysieren unter anderem die Umwandlung von Arachidonsäure, einer ungesättigten Fettsäure, die über verschiedene weitere Schritte zur Bildung von Prostaglandinen bzw. Leukotrienen führt. Diese haben wiederum differenziell zumeist proentzündliche Wirkungen, so z.B. Prostaglandin E2 und Prostacyclin.

COX wird durch nichtsteroidale Antirheumatika wie Diclofenac oder Ibuprofen gehemmt. Aber auch zahlreiche Pflanzeninhaltsstoffe können auf COX bzw. LOX im Menschen einwirken [15]. Nachgewiesen wurde dieses Prinzip beispielsweise für Gamma-Linolensäure in Nachtkerzenöl, Borretschsamen und den Samen schwarzer Johannisbeeren, für Extrakte aus dem indischen Weihrauch (Boswellia serrata), aus der Teufelskralle (Harpagophytum procumbens), Mutterkraut (Tanacetum parthenium) und Weidenrinde [12]. 


\section{Zusammenfassung}

Bei entzündlichem Rheuma oder Arthrose hat sich der Einsatz von Phythotherapeutika bewährt. Die pflanzlichen Arzneimittel werden äußerlich, aber auch innerlich oder subkutan angewandt. Brennnessel, Borretschsamen, Teufelskralle oder Weidenrinde gehören zu den effektivsten Wirkstoffen. Erste Studien belegen ihre Wirksamkeit.

Ein weiteres, noch ursprünglicheres, da in der Signaltransduktionskaskade vorgängiges Wirkprinzip ist die Hemmung des nukleären Transkriptionsfaktors NFkappaB, der auch die COX- und LOX-Aktivierung triggert. Es wurde z.B. an Extrakten der Arnika, des Mutterkrautes und des Krallendorns (Uncaria tomentosa) nachgewiesen. Die Hemmung von Tumornekrosefaktor-alpha (TNF-alpha) ist ein etabliertes Therapieprinzip der konventionellen Rheumatologie, da TNF-alpha für die Aufrechterhaltung von Entzündungen eine wesentliche Rolle spielt. Es wurden daher auch Pflanzenextrakte daraufhin untersucht, und an verschiedenen Zellen wurde eine verminderte Sekretion von TNF-alpha unter anderem bei Extrakten aus Ashwaganda (Whitania somnifera), Mutterkraut und Krallendorn gezeigt [12]. Für die Brennnessel fand sich neben einer Reduktion der TNF-alpha-Sekretion auch eine Reifungshemmung dendritischer Zellen, was vermutlich bei Patienten mit rheumatoider Arthritis eine verringerte T-Zellaktivierung bewirken könnte [12, 5].

Da Phytotherapeutika Vielstoffgemische sind, finden sich häufig mehrere Wirkprinzipien gleichzeitig. Diese Polyfunktionalität kann, zumindest theoretisch, synergistische oder überadditive Effekte begründen. Derzeit sind die Phytotherapeutika jedoch unter diesem Aspekt präklinisch kaum und klinisch noch nicht untersucht.

\section{Phytotherapeutika sind Vielstoffgemi- sche. Daher finden sich in ihnen häufig mehrere Wirkprinzipien gleichzeitig.}

\section{Studienlage}

Insgesamt ist die Wirksamkeit der bisher in Deutschland verfügbaren Phytotherapeutika sowohl von der Studienlage als auch von der klinischen Erfahrung her bei der Arthrose als moderat und bei der rheumatoiden Arthritis als eher gering einzuschätzen. Sie ist nach 


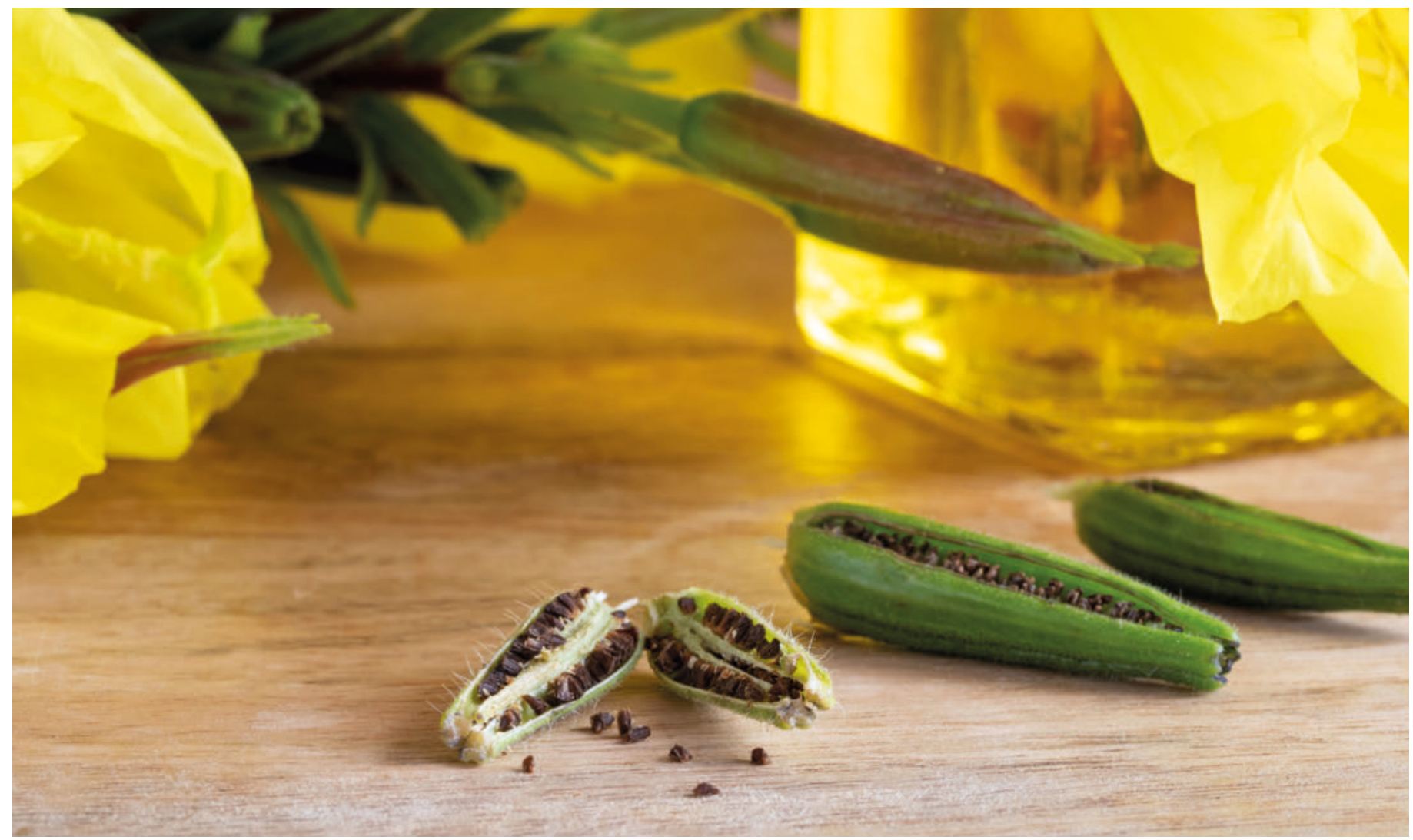

In Studien konnte Nachtkerzenöl die Symptome bei rheumatoider Arthritis bessern. Foto: Madeleine Steinbach/Adobe Stock

den Erfahrungen des Autors bei der rheumatoiden Arthritis zumeist deutlich geringer als die Wirkung der veganen Kost.

\section{Äußere Anwendung}

An pflanzlichen Externa werden bei Knie- oder Handarthose häufig Arnika (z. B. als Salbe 30\%, Gel oder Essenz) und Beinwell (z. B. Kyt$\mathrm{ta}^{\circledR}$ Salbe f, Traumaplant ${ }^{\circledR}$ Creme) eingesetzt. Sowohl für Arnika als auch für Beinwellpräparate besteht ein Wirksamkeitsnachweis durch einzelne placebokontrollierte, randomisierte Studien [1]. Beim entzündlichen Rheuma besteht erfahrungsgemäß ebenfalls eine topische Wirksamkeit. Studien hierzu gibt es bisher jedoch nicht. Capsaicin hemmt die Freisetzung von Entzündungsmediatoren (Substanz P) aus Nervenendigungen der Haut und hat dadurch analgetische Wirkungen. Sie werden typischerweise bei Nervenschmerzen eingesetzt, kommen aber auch bei Schmerzen durch Arthrose oder entzündliches Rheuma zum Tragen.

Bei Hand- und Kniegelenksarthrose war die Anwendung von Capsaicin 0,075\% in Studien wirksam $[13,8]$ und hat sich in Konzentrationen von 0,025-0,075\% (z.B. Capsagamma ${ }^{\circledR}$ Dolor 0,05\%, 3-4-mal tgl. dünn auftragen) bewährt. Bei Schmerzen durch entzündliches Rheuma finden sich keine Studien. Jedoch kommt es auch hier, insbesondere bei ausgebrannter Entzündung, erfahrungsgemäß zu einer schmerzlindernden Wirkung. Capsaicin darf nur bei intakter Haut angewendet werden und führt initial zu einer leichten Hautreizung.

\section{Innere Anwendung}

\section{Arthrose}

Bei der innerlichen Anwendung gibt es bei Arthroseschmerzen großer Gelenke positive Studienergebnisse für Teufelskralle, Hagebuttenpulver, Phytodolor und Weidenrinde [3]. Teufelskrallenextrakte soll- ten als standardisierte Fertigpräparate in ausreichender Dosierung ( $\geq 2400 \mathrm{mg}$ /Tag) eingenommen werden, da nur dann eine Wirkung zu erwarten ist. Die Behandlung wirkt nicht sofort, sondern erfordert im Allgemeinen 2-4 Wochen bis zur optimalen Wirkung. Hagebuttensamenpulver war in einer Dosierung von $5 \mathrm{~g} /$ Tag über 3 Monate zur Linderung von Beschwerden durch Hüft- und Kniearthrose effektiv [16]. Präparate, die Brennnessel, Weidenrinde und Phytodolor, eine Mischung aus Goldrutenkraut, Eschenrinde und Zitterpappelblätter und -rinde, enthalten, sind nach der Erfahrung und weniger aussagekräftigen Studien in hohen Dosierungen ebenfalls effektiv.

\section{Rheumatoide Arthritis}

Bei der rheumatoiden Arthritis findet sich aus kontrollierten, randomisierten Studien eine moderate Evidenz, dass die Symptome mit Nachtkerzenöl, Borretschöl oder Öl aus den Kernen der schwarzen Johannisbeere gebessert werden können [2]. Für die Wirksamkeit entscheidend ist hierbei der Gehalt an Gamma-Linolensäure. Nur bei höheren Dosen (äquivalent 1400 mg Gamma-Linolensäure) fanden sich therapeutische Effekte [3]. Für Präparate, die Brennnessel, Teufelskralle und Boswellia serrata enthalten, besteht nach der Erfahrung und einzelnen Studien ebenfalls eine gewisse Wirksamkeit zur Symptomlinderung. Brennnessel kann auch als Gemüse blanchiert oder gedünstet eingenommen werden und führte nach Erfahrungen des Autors in Einzelfällen bei Patienten, die dies umsetzten, zu deutlichen Verbesserungen der Symptome.

Mutterkrautextrakt war in einer placebokontrollierten Studie bei rheumatoider Arthritis nicht wirksam [11]. Ein Präparat aus dem Krallendorn (Uncaria tomentosa, 3-mal tgl. 20 mg KrallendornKapseln, frei von tetrazyklischen Oxindolalkaloiden) führte in einer kleinen Studie zu einer Reduktion der Anzahl schmerzhafter Gelenke im Vergleich zum Placebo. Die anderen Parameter - Anzahl der 
geschwollenen Gelenke, Schmerzen, Morgensteifheit, C-reaktives Protein - besserten sich jedoch nicht gegenüber dem Placebo [10].

\section{Verträglichkeit}

Die Verträglichkeit der genannten Phytotherapeutika ist im Allgemeinen sehr gut. Bei den für die Therapie erforderlichen höheren Dosierungen kann es in Einzelfällen zu Magen-Darm-Beschwerden kommen. Teufelskralle hat wahrscheinlich keinen Einfluss auf die Funktion von Cytochrom-Peroxidasen [9] und damit kein relevantes Interaktionsrisiko, während Boswelliaextrakte einen hemmenden Einfluss auf Cytochrom P 450 haben können [6].

Neben diesen klassischen Phytotherapeutika gibt es pflanzliche Mittel der Anthroposophischen Medizin zur oralen (z. B. Colchicum tuber, Mandragora officinale), lokalen (z.B. Aconitöl 5\%) oder parenteralen, zumeist subkutanen oder periartikulären Anwendung (z.B. Equisetum arvense, Betula pendula, Mandragora, Solum uliginosum, Viscum album) in verschiedenen sowohl homöopathischen wie substanziellen Dosierungen. Insbesondere die Therapie mit Viscumpräparaten ist ein interessantes Prinzip, da diese bei subkutaner Injektion eine T-Helfer-2-vermittelte Immunantwort auslösen [7], was bei einer T-Helfer-1-zellvermittelten Autoimmunerkrankung wie der rheumatoiden Arthritis eine therapeutische Immunmodulation bewirken könnte. Hierzu gibt es aber bisher keine Studien.

Nach der Erfahrung können Mistelpräparate lokal über den betroffenen Gelenken (Cave: nicht in das Gelenk) injiziert als Reiztherapie zur Behandlung von Arthroseschmerzen gut eingesetzt werden. Ein in der Anthroposophischen Medizin parenteral angewendeter Birkenblätterextrakt (Abnoba Betula Folium D3) hatte in vitro und in einem Tierversuch ausgeprägte antientzündliche Eigenschaften [14]. Letztlich sind viele auch europäische Pflanzen noch nicht in ihrer Wirkung auf das Immunsystem untersucht, und es ist zu erwarten, dass weitere mit Potenzial zur Behandlung von Rheuma gefunden werden.

Interessenkonflikt: Der Autor erklärt, dass keine persönlichen oder wirtschaftlichen Interessen bestehen.

\section{Online zu finden unter \\ http://dx.doi.org/10.1055/a-0995-6076}

\section{Erstveröffentlichung}

Verband für Ernährung und Diätetik e.V. Die richtige Ernährung bei entzündlichem Rheuma und Gicht. 2015; 17-19; V VFED

Der Artikel wurde für die vorliegende Fassung aktualisiert.

\footnotetext{
Literatur

1 Cameron M, Chrubasik S. Topical herbal therapies for treating osteoarthritis. Cochrane Database Syst Rev 2013; 5: CD010538

2 Cameron M, Gagnier J], Chrubasik S. Herbal therapy for treating rheumatoid arthritis. Cochrane Database Syst Rev 2011; 2: CD002948

$\overline{3}$ Cameron M, Gagnier J], Little CV et al. Evidence of effectiveness of herbal medicinal products in the treatment of arthritis. Part I: Osteoarthritis. Phytother Res 2009; 23(11): 1497-1515

4 Cameron M, Gagnier J], Little CV et al. Evidence of effectiveness of herbal medicinal products in the treatment of arthritis. Part 2: Rheumatoid arthritis. Phytother Res 2009; 23(12): 1647-1662

5 Dragos D, Gilca M, Gaman L et al. Phytomedicine in joint disorders. Nutrients 2017; 9(1): 70
}

6 Frank A, Unger M. Analysis of frankincense from various Boswellia species with inhibitory activity on human drug metabolising cytochrome P450 enzymes using liquid chromatography mass spectrometry after automated online extraction. J Chromatogr A 2006; 1112(1-2): 255-262

7 Huber R, Rostock M, Goedl R et al. Mistletoe treatment induces GM-CSFand IL-5 production by PBMC and increases blood granulocyte- and eosinophil counts: A placebo controlled randomized study in healthy subjects. Eur ] Med Res 2005; 10(10): 411-418

8 McCarthy GM, McCarty DJ. Effect of topical capsaicin in the therapy of painful osteoarthritis of the hands. J Rheumatol 1992; 19(4): 604-607

9 Modarai M, Suter A, Kortenkamp A et al. The interaction potential of herbal medicinal products: A luminescence-based screening platform assessing effects on cytochrome P450 and its use with devil's claw (Harpagophyti radix) preparations. J Pharm Pharmacol 2011; 63(3): 429-438

$\overline{10}$ Mur E, Hartig F, Eibl G et al. Randomized double blind trial of an extract from the pentacyclic alkaloid-chemotype of uncaria tomentosa for the treatment of rheumatoid arthritis. J Rheumatol 2002; 29(4): 678-681

$\overline{11}$ Pattrick M, Heptinstall S, Doherty M. Feverfew in rheumatoid arthritis: A double blind, placebo controlled study. Ann Rheum Dis 1989; 48(7): 547549

$\overline{12}$ Setty AR, Sigal LH. Herbal medications commonly used in the practice of rheumatology: Mechanisms of action, efficacy, and side effects. Semin Arthritis Rheum 2005; 34(6): 773-784

$\overline{13}$ Schnitzer T], Pelletier JP, Haselwood DM et al. Civamide cream $0.075 \%$ in patients with osteoarthritis of the knee: A 12-week randomized controlled clinical trial with a longterm extension. J Rheumatol 2012; 39(3): 610-620

$\overline{14}$ Wacker K, Gründemann C, Kern Y et al. Inhibition of corneal inflammation following keratoplasty by birch leaf extract. Exp Eye Res 2012; 97(1): 24-30

$\overline{15}$ Werz 0 . Inhibition of 5-lipoxygenase product synthesis by natural compounds of plant origin. Planta Med 2007; 73(13): 1331-1357

$\overline{16}$ Winther K, Apel K, Thamsborg G. A powder made from seeds and shells of a rose-hip subspecies (Rosa canina) reduces symptoms of knee and hip osteoarthritis: A randomized, double-blind, placebo-controlled clinical trial. Scand J Rheumatol 2005; 34(4): 302-308

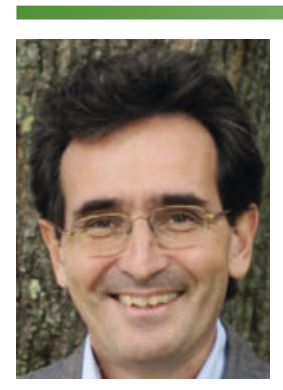

Prof. Dr. Roman Huber

Uni-Zentrum Naturheilkunde

Universitätsklinikum Freiburg

Breisacher Str. $115 b$

79106 Freiburg

roman.huber@uniklinik-freiburg.de

Prof. Dr. Roman Huber studierte Medizin und promovierte am Institut für Arbeitsphysiologie und Rehabilitationsforschung der Philipps-Universität Marburg. Seither ist er in verschiedenen Krankenhäusern als Arzt tätig und führt als Facharzt für Innere Medizin mit dem Schwerpunkt Gastroenterologie die Zusatzbezeichnung Naturheilverfahren. Seit 2003 ist er Oberarzt am Universitätsklinikum Freiburg und leitet das Uni-Zentrum Naturheilkunde. 